Die „MilbenCheck“-App

\section{Mobile Hilfe für Milbenallergiker}

\author{
Nach dem Vorbild der Smartphone-App „Pollen“ (Allergo Journal \\ $3 / 2014$, S. 66) hat die Stiftung Deutscher Polleninformationsdienst in \\ Zusammenarbeit mit der Forschungsgruppe Aerobiologie und \\ Polleninformation an der Medizinischen Universität Wien auch für \\ Milbenallergiker eine App entwickelt: Die „MilbenCheck“-App.
}

$\mathrm{D}$ ie Hausstaubmilbenallergie zählt mit zu den häufigsten in Deutschland vorkommenden Allergien. So leiden z. B. auch $30 \%$ der Pollenallergiker an einer Allergie gegen Hausstaubmilben. Vor diesem Hintergrund ging vor kurzem das Portal www.milbencheck.de online. Ergänzend dazu gibt es eine mobile Applikation für Milbenallergiker: Die Smartphone-App „MilbenCheck“ bietet mit einem Risikofragebogen, einem Tagebuch und einer Milbenbelastungslandkarte Unterstützung für Betroffene.

\section{Milbenallergie rechtzeitig erkennen}

Der neue Service „MilbenCheck“ unterstützt und begleitet Milbenallergiker auf wissenschaftlich fundierter Basis und berücksichtigt dabei die individuelle Situation des Betroffenen. Sowohl die App als auch das dazu passende Online-Portal besitzen eine einfache Menüführung und klar strukturierte Kapitel. Sie unterstützen den Anwender dabei, die Allergie im täglichen Leben, am Arbeitsplatz oder in der Schule zu dokumentieren, besser zu verstehen und damit umzugehen. Das Ziel dabei ist es, die Erkrankung frühzeitig zu erkennen, zur Abklärung der Symptome einen Besuch bei einem allergologisch geschulten Facharzt anzuregen und eine rasche Therapie zu ermöglichen.

\section{Risikofragebogen und Milbentagebuch}

Ein einfacher Selbsttest bietet eine erste Einschätzung, ob eine Milbenallergie für die vorliegenden Beschwerden verantwortlich sein kann. Die 14 Fragensets lassen sich rasch beantworten und sie ordnen den Nutzer einer von vier Risikogruppen zu: Ein erster Schritt, der die Entscheidung zur Symptomabklärung bei einem allergologisch geschulten Facharzt unterstützen soll. Der Online-Selbsttest liefert für die weitere Diagnose wichtige Informationen, die als E-Mail versendet oder als Papierausdruck zum Arzttermin mitgenommen werden können.

Eine weitere Funktion der App ist das „Milbentagebuch“. Durch regelmäßige Einträge des Nutzers entsteht damit ein individuelles Beschwerdeprofil. Die eingetragenen Daten stehen auf Knopfdruck als Grafik zur Verfügung, schaffen ein individuelles Gesamtbild der Allergie und können auch eine Unterstützung für die behandelnden Ärzte sein.

\section{Die Milbenbelastungslandkarte}

Die Milbenbelastungslandkarte macht es möglich, die eigenen Beschwerden mit denen anderer Allergiker in der Umgebung zu vergleichen, und hilft, die persönliche Allergiesituation einzuschätzen. Die Symptom-Landkarte visualisiert die Symptomdaten aller Tagebuchnutzer anonymisiert auf einer Europakarte und gibt so einen Überblick über die Beschwerdeintensität anderer Milbenallergiker.

Auch ein persönlicher Erinnerungsservice bietet als Teil der App Hilfe im Allergiker-Alltag. Der Nutzer wird nach der Anmeldung viermal im Jahr auf einen Facharztbesuch aufmerksam gemacht.

Mit „MilbenCheck“ stehen nun auch Milbenallergikern ein Portal und eine App zur Verfügung, die aktiv begleiten, die Diagnose erleichtern und einen klaren Überblick über die Beschwerden und den Therapieverlauf schaffen können. Die neue Applikation für iOS- und Android-Geräte steht zum kostenlosen Download zur Verfügung. Das zugehörige Online-Portal ist unter der Adresse www.milbencheck.de zu finden. red

\section{CK-CARE stellt sich neu auf}

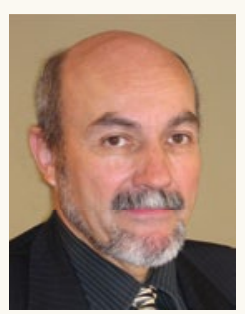

Prof. Dr. Dr. Johannes Ring, Dir. emer. Klinik und Poliklinik für Dermatologie und Allergologie am Biederstein, Klinikum rechts der Isar, Technische Universität München, ist altersbedingt aus dem Direktorium des "Christine Kühne - Centers for Allergy Research and Education" (CK-CARE) ausgeschieden. Er wird CK-CARE aber weiterhin als Consultant in der Edukation zur Verfügung stehen.

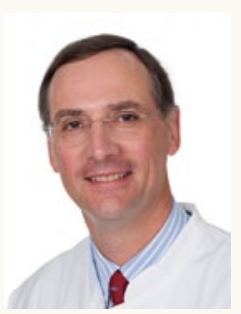

Neu im CK-CAREDirektorium ist Prof. Dr. Dr. Thomas Bieber, Klinik und Poliklinik für Dermatologie und Allergie, Universität Bonn. Er hat die Leitung des Workpackage 3 „Remission und Immuntoleranz bei atopischen Erkrankungen“ übernommen.

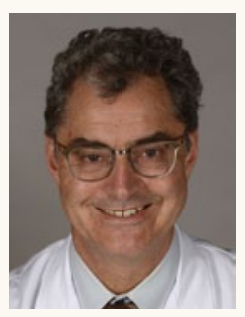

Ebenfalls neu im Direktorium ist Prof. Dr. Peter Schmid-Grendelmeier, Allergiestation des Universitätsspitals Zürich, Schweiz. Er hat die Leitung des Workpackage 5 „Schulung als Brücke zwischen Patient und Forschung" übernommen.

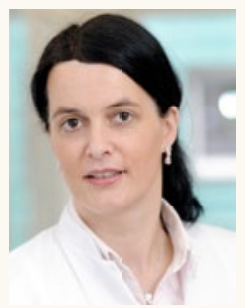

Direktoriumsmitglied Prof. Dr. Claudia Traidl-Hoffmann, leitet weiterhin das Workpackage 1 „Die Einflüsse der Umwelt auf atopische Erkrankungen", hat aber außerhalb von CK-CARE eine neue Herausforderung angenommen: Sie ist seit 1. Oktober Chefärztin für Umweltmedizin am Klinikum Augsburg.

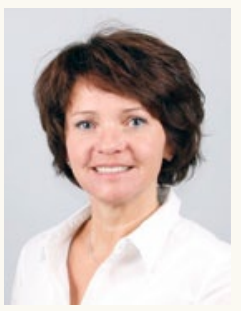

Die Leitung des CK-CARE-Sekreteriats, Geschäftsstelle Davos, hat Jacqueline Boller übernommen. 